\title{
A Method for Service-Oriented Personalized Requirements Analysis
}

\author{
Huafeng Chen, Keqing He
}

State Key Laboratory of Software Engineering, Wuhan University, Wuhan, China

Email: canada186771@yahoo.com.cn, hekeqing@public.wh.hb.cn

Received November $22^{\text {nd }}, 2010$; revised December $4^{\text {th }}$, 2010; accepted December $20^{\text {th }}, 2010$.

\begin{abstract}
The development of Web service has changed the process of software production, and requirements engineering becomes the key issue of service-oriented software engineering. Meantime, it reduces the degree of difficulty of software production, which facilitates end-users to customize software according to their personalized requirements. The paper proposes a method for service-oriented personalized requirements analysis, which is based on domain goal model and process model. The method can inform users of potential errors in requirements by detecting the correctness of requirements, which is driven by users' personalized operations on goal models, and customize personalized processes to satisfy users' requirements by reusing domain processes. The personalized processes are the basis for Web service discovery and composition.
\end{abstract}

Keywords: Personalized Requirements Analysis, Requirements Correctness Detection, Personalized Process Customization, Domain Model, Web Service

\section{Introduction}

The development of Web service has changed the process of software production. Traditionally, the process of software production includes several phases, such as requirements elicitation and analysis, design, coding and test. However, in the era of service computing, more and more Web services are deployed on the Internet, which provide plenty of resources for software development and facilitate a novel software production methodology "Meet-in-the-Middle" [1]. This novel methodology reduces the degree of difficulty of software production and makes it possible for end-users with some knowledge about computer to customize their own software according to their personalized requirements. The software is composed of Web services and can be changed easily according to the requirements. Because the software is living on the Internet, we name it networked software [2-6].

Using existing Web services is the basis for customizing networked software, so the key problem to solve is how to get accurate requirements from end-user, rather than design and coding.

To serve the end-user well, we think a tool to support the procedure of customization is necessary. The tool should give them a lot of tips during the customization, since end-users are always without expert knowledge about software, but some knowledge about what they want to do. These tips should come from domain models which are constructed by experts beforehand.

Domain models usually include common requirements and solutions, but requirements from end-users are always personalized. The paper focuses on how to analyze end-users' personalized requirements using common requirements in domain models.

The paper is organized as follows. Section 2 is about related works. In Section 3, the RGPS requirements meta-model framework is introduced, which is the guideline of constructing domain models used in requirements analysis. In Section 4, we introduce how to analyze serviceoriented personalized requirements based on domain models. The procedure of analysis includes detecting the correction of personalized requirements and personalized process customization. A case study is illustrated in Section 5. A prototype tool based on our method is introduced in Section 6. The last section concludes the paper and proposes some problems should be solved in the future.

\section{Related Works}

Our method in this paper is related to several ideas or methodologies, such as mass customization, goal-orien- 
ted requirements analysis, and techniques for personalized process customization.

The idea of mass customization is successfully applied in software engineering. One proof is the birth of software product line, which is related to domain analysis and modeling. Constructing domain requirements assets is usually necessary. Feature-oriented domain analysis [7] is very useful to construct common requirements and variable requirements [8]. The decomposition of goals in RGPS meta-model adopts similar idea.

Goal-oriented requirements analysis is popular after object-oriented requirements analysis [9]. It is more suitable for people without expert knowledge to describe requirements. KAOS [10] and Tropos [11] are classical goal-oriented requirements analysis methods.

In the era of service computing, goal-oriented requirements analysis is still full of vitality. Tropos is extended to make it possible to get service specification [12].

Personalized process customization is the key technique in service computing. A Web service customization model is constructed in [13], which is related to process customization. Web service customization is achieved by applying goal ontology model, process component reuse and flexible process defining. The process is described with ECA [14], and users are allowed to modify process directly.

One of the characteristics of our work is that we face end-user. Service Oriented Architectures for All (SOA4All) is a large-scale integrating project funded by the European Seventh Framework Programme, under the Service and Software Architectures, Infrastructures and Engineering research area [15], which also faces enduser. The objective of SOA4All is to make the service Web as accessible and ubiquitous as today's information Web. It aims at integrating SOA and four complementary and revolutionary technical advances (the Web, contextaware technologies, Web 2.0 and Semantic Web) into a coherent and domain independent worldwide service delivery platform.

\section{RGPS Requirements Meta-Model Framework}

The RGPS requirements meta-model framework adopts ontology \& meta-modeling theory [16], and defines 4 types of elements required by service-oriented requirements modeling. They are role, goal, process and service, res- pectively. The role model and the goal model are des- cribed in OWL [17], and the process model and the service model are described in OWL-S [18]. All the models are annotated with ontology, which makes it possible for semantic inquiry and reasoning. 4 types of elements are not isolated. A role can have goals, a goal can be achieved by processes, and a process can be rea- lized by services.

This requirements meta-model framework can be used to guide domain experts to construct domain models. The common requirements assets in domain models can be used to analyze requirements and customize personalized process.

For the goal and the process are greatly related to this paper, we will look further into them. For the comprehensive understanding of RGPS, we can refer to [19].

\subsection{Goal Meta-Model}

Goals and relationships between goals are two major elements in the goal meta-model (Figure 1). Goals are defined as the target state of the system users expect, and are divided into functional goals and nonfunctional goals. Functional goals have 3 properties, and they are operation, such as "display", object, such as "travel information", and manner, such as "by SMS”. Nonfunctional goals are further divided into qualitative nonfunctional goal and quantitative nonfunctional goal. The former, such as "fast responsible time", includes nonfunctional type, such as "responsible time", and degree, such as "fast". The latter, such as "cost less than 20 dollars", includes nonfunctional type, such as "cost”, comparison operator, such as "less", value, such as “20”, and unit, such as “dollar”. A nonfunctional goal can affect one functional goal or the whole system.

The relationship between goals can be divided into horizontal relationship and vertical relationship. The former include "depend" and "exclude”. The “depend” relationship means that if the goal A is selected, the goal B which is depended by the goal A, should be selected. The goal $\mathrm{A}$ is the source end, and the goal B is the target end. The "exclude" relationship means that if a goal is selected, all the goals exclude it should not be selected.

The vertical relationship between goals is "decompose". Goals can be refined by decomposition until operational goals which can be achieved by processes. We define 4 types of decomposition, and they are "mandatory”, "optional", "alternative” and "or". The "mandatory" means that if the super goal is selected, the sub-goal should be selected. The "optional" means that if the super goal is selected, the sub-goal could be selected or not. The "alternative" means that if the super goal is selected, one of the sub-goals in a group should be selected. The "or" means that if the super goal is selected, at least one of the sub-goals in a group should be selected.

\subsection{Process Meta-Model}

The process meat-model includes process and its properties (Figure 2). The process can be divided into atomic process and composite process. Atomic processes are composed into a composite process by control structures. 


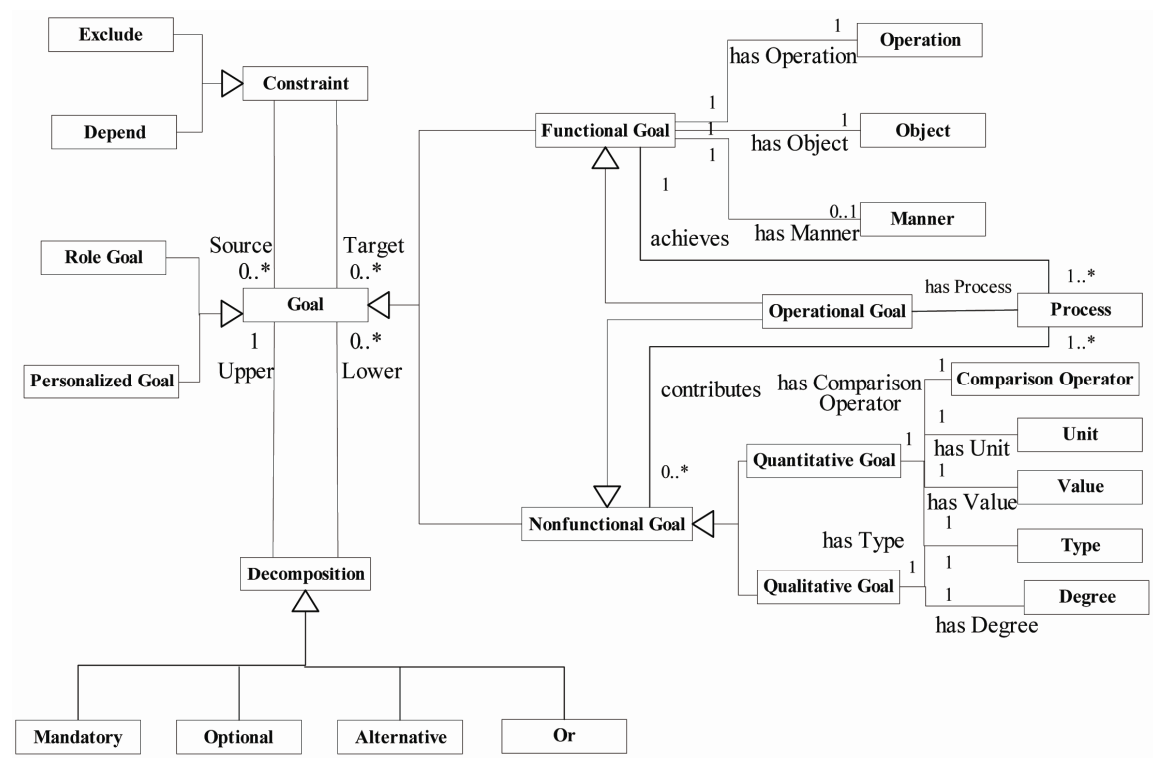

Figure 1. Goal meta-model.

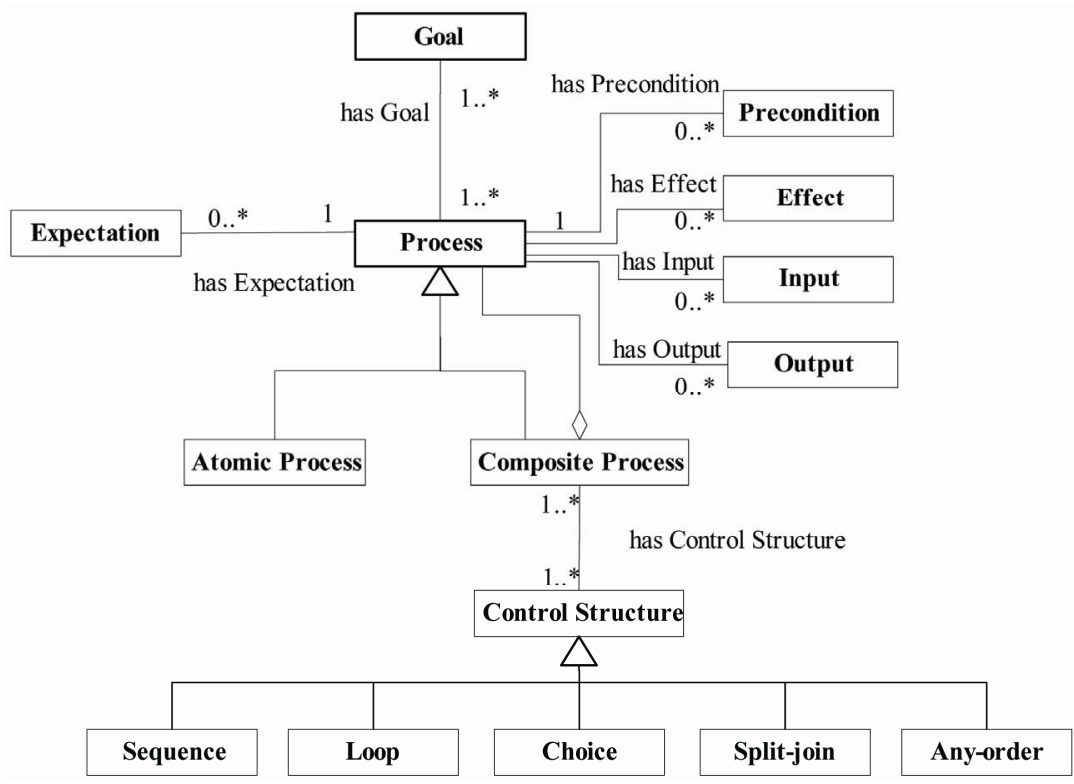

Figure 2. Process meta-model.

5 typical control structures are in the meta-model. They are sequence, loop, choice, split-join and any-order. The properties of process include input, output, precondition, effect, and quality expectation. The input and output represent data flow of the process. The precondition and effect represent the condition must be satisfied before execution and after execution, respectively. The quality expectation means nonfunctional constraint on the process.

\subsection{Relationship between Goal and Process}

The relationships between goal and process are also de- fined in the framework. Due to the limitation of space, we illustrate them in Table 1. More details are in [20].

Table 1. Relationship between Goal and Process.

\begin{tabular}{ll}
\hline Goal & Process \\
\hline Functional Goal & Composite Process \\
Operational Goal & Atomic Process \\
Mandatory & Any-Order \\
Optional & Any-Order \\
Alternative & Choice \\
Or & Split-Join \\
Depend & Sequence \\
Nonfunctional Goal & Expectation \\
\hline
\end{tabular}




\section{A Method for Service-Oriented Personalized Requirements Analysis}

The method proposed in the paper allows end-users to propose their personalized requirements by modifying domain goal models. We name this type of modification personalized operation. After the personalized operation, different types of requirements correctness detections will be triggered. The modifications on domain goal models will be mapped into process models and personalized processes are generated automatically by modifying domain process models. Because end-users are more familiar with the goals than processes, we advocate this method is suitable for end-users to customize software.

\subsection{The Relationship between Personalized Operations and Correctness Detection}

Because the requirements in domain models are common, but end-users' requirements are personalized, there must be some difference between them. However, some of the difference is problematic. The task of correctness detection is to find the problematic difference. Table 2 illustrates corresponding correctness detections to personalized operations.

\subsection{Personalized Operations and Correctness Detection}

In this paper, personalized operations are divided into 2 categories. One category will introduce requirements from other domain into the current requirements, whereas the other category won't. The former is named interdomain personalized operation, and the latter is named personalized operation within one domain.

Personalized operation within one domain can be divided into 8 sub-categories, and they are "Select Subgoal”, "Specify Goal”, “Add Domain Functional Goal”, "Delete Domain Functional Goal”, “Add Nonfunctional Goal within a Domain”, "Delete Nonfunctional Goal within a Domain”, “Add 'Depend' between Goals within a Domain", and "Delete 'Depend' within a Domain”. Inter-domain personalized operation can be divided into 6 sub-categories, and they are "Add Functional Goal of other Domain”, "Delete Functional Goal of other Domain”, “Add Nonfunctional Goal of other Domain”, "Delete Nonfunctional Goal of other Domain”, "Add 'Depend' between Goals not within a Domain”, and “Delete 'Depend' between Goals not within a Domain”.

\subsubsection{Personalized Operations within One Domain}

\section{1) Select Sub-goal (SSG)}

Selecting a sub-goal is the most common operation for end-users. They should follow 4 types of decompositions.
By the way, the selected goal may be excluded by existing goals. So "Check Decomposition Conflict" and "Check Conflict” are necessary.

"Check Decomposition Conflict" means checking whether end-users' selections for sub-goals should follow the constraints of 4 types of decomposition.

"Check Conflict" means checking whether the subgoal selected by end-user has the "exclude" relationship between other goals existing in the requirements.

\section{2) Specify Goal (SG)}

Specifying a goal means adding the "manner" to the goal. For example, the goal "display travel information", which does not have "manner", can be specified into “display travel information by SMS”, by adding "manner” "by SMS”. Specifying a goal won't cause any trouble, so correctness detection is unnecessary.

3) Add Domain Functional Goal (ADFG)

A functional goal can be added as a sub-goal of an existing goal or as a top-level goal. A special situation is that if goal A is selected and goal A depends on goal B in the domain model, the end-user can add goal $B$ together with the "depend" relationship. In this case, goal B is called "associated goal". "Check Conflict” is necessary in this situation. If a goal is added without any "depend" relationship, we must do "Check Sub-goal”, and "Check Conflict".

"Check Sub-goal” means checking whether a goal can be a sub-goal of another goal. We assume that a goal in the domain model can not be the super-goal of its former super-goal or can not be the sub-goal of its former subgoal.

\section{4) Delete Domain Functional Goal (DDFG)}

When deleting a goal with the "depend" relationship, we should do "Check Dependent Goal Collection", "Check Nonfunctional Goal Collection”, and "Check Subgoal Collection". When deleting a goal without the "depend" relationship, we should only do "Check Nonfunctional Goal Collection”, and "Check Sub-goal Collection”.

"Check Dependent Goal Collection" means that we should delete the goal which is depended by the goal deleted.

“Check Nonfunctional Goal Collection” means that the nonfunctional goals, which constrain the functional goal deleted, should be deleted.

"Check Sub-goal Collection" means that we should delete the sub-goals of the goal deleted.

5) Add Nonfunctional Goal within a Domain (ANFGD)

Adding a nonfunctional goal to a functional goal will trigger "Check Nonfunctional Type". Because the nonfunctional goal may conflict with the nonfunctional goals already related to the same functional goal.

6) Delete Nonfunctional Goal within a Domain (DNFGD) 
Table 2. Relationship among personalized operation, correctness detection and personalized process customization.

\begin{tabular}{|c|c|c|c|c|c|}
\hline \multicolumn{3}{|c|}{ Personalized Operation } & Correctness Detection & $\begin{array}{l}\text { Personalized Process } \\
\text { Customization }\end{array}$ & Note \\
\hline \multirow{12}{*}{$\begin{array}{l}\text { Personalized } \\
\text { Requirements } \\
\text { Within One } \\
\text { Domain }\end{array}$} & \multirow{3}{*}{$\begin{array}{l}\text { Select Sub-goal } \\
\text { (SSG) }\end{array}$} & $\begin{array}{c}\text { Select Optional } \\
\text { Sub-goal (SOSG) }\end{array}$ & \multirow{3}{*}{$\begin{array}{l}\text { Check Decomposition } \\
\text { Conflict (C_DC), Check } \\
\text { Conflict(C_C) }\end{array}$} & $\begin{array}{l}\text { Delete Process in } \\
\text { Any-order (DPA) }\end{array}$ & \\
\hline & & $\begin{array}{l}\text { Select Alternative } \\
\text { Sub-goal (SASG) }\end{array}$ & & $\begin{array}{l}\text { Delete Process in Choice } \\
\text { (DPC) }\end{array}$ & \\
\hline & & $\begin{array}{l}\text { Select Or } \\
\text { Sub-goal } \\
\text { (SORSG) }\end{array}$ & & $\begin{array}{l}\text { Delete Process in } \\
\text { Split-join (DPS) }\end{array}$ & \\
\hline & \multicolumn{2}{|c|}{ Specify Goal (SG) } & No & Add Input (AI) & $\begin{array}{l}\text { Specify a goal by } \\
\text { adding "manner" } \\
\text { to the goal. }\end{array}$ \\
\hline & \multirow{2}{*}{$\begin{array}{l}\text { Add Domain } \\
\text { Functional Goal } \\
\text { (ADFG) }\end{array}$} & $\begin{array}{l}\text { By “Depend” } \\
\text { (ADFGD) }\end{array}$ & Check Conflict(C_C) & $\begin{array}{l}\text { Add Process Considering } \\
\text { Dependency (APD) }\end{array}$ & \multirow{2}{*}{$\begin{array}{l}\text { A way to complete } \\
\text { requirements. }\end{array}$} \\
\hline & & $\begin{array}{l}\text { Not by "Depend" } \\
\text { (ADFGND) }\end{array}$ & $\begin{array}{l}\text { Check Sub-goal (C_S), } \\
\text { Check Conflict(C_C) }\end{array}$ & $\begin{array}{l}\text { Add Any-order and a } \\
\text { Process in Any-order } \\
\text { (APA) }\end{array}$ & \\
\hline & \multirow{2}{*}{$\begin{array}{l}\text { Delete Domain } \\
\text { Functional Goal } \\
\text { (DDFG) }\end{array}$} & $\begin{array}{l}\text { With "Depend" } \\
\text { (DDFGD) }\end{array}$ & $\begin{array}{c}\text { Check Dependent Goal } \\
\text { Collection (C_DGClct), } \\
\text { Check Nonfunctional } \\
\text { Goal Collection } \\
\text { (C_NFGClct), Check } \\
\text { Sub-goal Collection } \\
\text { (C_SubClct) }\end{array}$ & \multirow[t]{2}{*}{ Delete Process (DP) } & $\begin{array}{l}\text { The goal deleted } \\
\text { has "depend" } \\
\text { relationship with } \\
\text { another goal. }\end{array}$ \\
\hline & & $\begin{array}{l}\text { Without "De- } \\
\text { pend" } \\
\text { (DDFGND) }\end{array}$ & $\begin{array}{l}\text { Check Nonfunctional } \\
\text { Goal Collection } \\
\text { (C_NFGClct), Check } \\
\text { Sub-goal Collection } \\
\text { (C_SubClct) }\end{array}$ & & $\begin{array}{l}\text { The goal deleted } \\
\text { does not have } \\
\text { “depend” rela- } \\
\text { tionship with } \\
\text { another goal. }\end{array}$ \\
\hline & \multicolumn{2}{|c|}{$\begin{array}{l}\text { Add Nonfunctional Goal within a } \\
\text { Domain (ANFGD) }\end{array}$} & $\begin{array}{l}\text { Check Nonfunctional } \\
\text { Type (C_NFT) }\end{array}$ & Add Expectation (AE) & \\
\hline & \multicolumn{2}{|c|}{$\begin{array}{l}\text { Delete Nonfunctional Goal within a } \\
\text { Domain (DNFGD) }\end{array}$} & No & Delete Expectation (DE) & \\
\hline & \multicolumn{2}{|c|}{$\begin{array}{l}\text { Add "Depend” between Goals within a } \\
\text { Domain (ADD) }\end{array}$} & Check Depend (C_D) & \multirow{2}{*}{ No } & \\
\hline & Delete "Depend & $\begin{array}{l}\text { within a Domain } \\
\text { D) }\end{array}$ & No & & \\
\hline \multirow{6}{*}{$\begin{array}{l}\text { Inter-domain } \\
\text { Personalized } \\
\text { Requirements }\end{array}$} & \multicolumn{2}{|c|}{$\begin{array}{l}\text { Add Functional Goal of other Domain } \\
\text { (AFG) }\end{array}$} & No & $\begin{array}{l}\text { Add any-order and a } \\
\text { Process in Any-order } \\
\text { (APA) }\end{array}$ & \\
\hline & \multicolumn{2}{|c|}{$\begin{array}{l}\text { Delete Functional Goal of other Do- } \\
\text { main (DFG) }\end{array}$} & The same as (DDFG) & Delete Process (DP) & \\
\hline & \multicolumn{2}{|c|}{$\begin{array}{l}\text { Add Nonfunctional Goal of other Do- } \\
\text { main (ANFG) }\end{array}$} & $\begin{array}{l}\text { Check Nonfunctional } \\
\text { Type (C_NFT) }\end{array}$ & Add Expectation (AE) & $\begin{array}{l}\text { The nonfunctional } \\
\text { goal must con- } \\
\text { strain a nonfunc- } \\
\text { tional goal, which } \\
\text { belongs to other } \\
\text { domain. }\end{array}$ \\
\hline & \multicolumn{2}{|c|}{$\begin{array}{l}\text { Delete Nonfunctional Goal of other } \\
\text { Domain (DNFG) }\end{array}$} & The same as (DDNFG) & Delete Expectation (DE) & \\
\hline & \multicolumn{2}{|c|}{$\begin{array}{l}\text { Add “Depend” between Goals not } \\
\text { within a Domain (AD) }\end{array}$} & Check Depend (C_D) & No & $\begin{array}{l}\text { The “depend” } \\
\text { relationship must } \\
\text { be related to a } \\
\text { goal, which be- } \\
\text { longs to other } \\
\text { domain. }\end{array}$ \\
\hline & \multicolumn{2}{|c|}{$\begin{array}{l}\text { Delete "Depend" between Goals not } \\
\text { within a Domain (DD) }\end{array}$} & The same as (DDD) & & \\
\hline
\end{tabular}

Deleting a nonfunctional goal won't cause any incorrectness.
7) Add "Depend" between Goals within a Domain (ADD) Adding a “depend” relationship between two goals will 
trigger "Check Depend”.

"Check Depend" means checking whether the "depend" relationship will become a loop after adding a new one.

8) Delete "Depend" within a Domain (DDD)

Deleting a "depend" relationship between two goals won't cause any incorrectness.

\subsubsection{Inter-domain Personalized Operations}

\section{1) Add Functional Goal of other Domain (AFG)}

Adding a functional goal belonging to another domain won't cause any incorrectness, because the goal from another domain does not have any relationship with goals in this domain.

\section{2) Delete Functional Goal of other Domain (DFG)}

Because once a goal from another domain is added into the existing requirements, the context of the goal is the same as goals in this domain, deleting the goal is the same as DDFG.

\section{3) Add Nonfunctional Goal of other Domain (ANFG)}

Adding a nonfunctional goal from another domain will trigger "Check Nonfunctional Type".

4) Delete Nonfunctional Goal of other Domain (DNFG)

Deleting a nonfunctional goal from another domain is the same as DNFGD.

5) Add "Depend" between Goals not within a Domain (AD)

"Check Depend" is necessary after adding "depend" relationship between goals from different domains.

6) Delete "Depend" between Goals not within a Domain (DD)

Deleting "depend" is the same as DDD.

\subsection{Personalized Process Customization}

Personalized process customization is driven by endusers' personalized operations. Having detecting correctness of requirements, we can modify domain process models to satisfy end-users' requirements using relationship between goal and process (Table 1) and rules for customizing processes. Table 2 illustrates the rules corresponding to personalized operations.

\section{1) Delete Process in Any-order (DPA)}

In Table 1, optional goals correspond to the processes in control structure "any-order" (Figure 3(a)). If the optional goals selected by end-users correspond to process $i$, process $\mathrm{j}$, and process $\mathrm{k}$, we can modify the common process (Figure 3(a)) into the personalized one (Figure 3(b)). When only one process existed in the control structure "any-order", it is unnecessary for it to exist, so we delete the control structure "any-order".

2) Delete Process in Choice (DPC)

The situation is similar to DPA. It is easily to under- stand the transformation from the process in Figure 3(c) to the process in Figure 3(d). It should be noticed that in this case only one goal is selected, so the control structure "choice" is always deleted.

3) Delete Process in Split-join (DPS)

This case is similar to DPA.

\section{4) Add Input (AI)}

"Manner" added by end-uses can be transformed into the input of the process. For example, if an end-user specifies a goal "search gas station" into "search gas station by current position”, the corresponding process will add an input "current position".

\section{5) Add Process Considering Dependency (APD)}

The "depend" relationship corresponds to the control structure "sequence". If goal i depends on goal $j$, and goal $j$ is added by end-user, we should transform process i (Figure 3 (d)) into the personalized one (Figure 3(g)).

6) Add Any-order and a Process in Any-order (APA)

When an end-user adds a goal without "depend" relationship with other goals, we should transform the process (Figure 3(d)) into the personalized one (Figure 3 (h)).

7) Delete Process (DP)

Delete the process which corresponding to the goal deleted by end-user.

\section{8) Add Expectation (AE)}

Transform the nonfunctional goal added by end-user into expectation. The nonfunctional goal is usually attached to a functional goal, so the expectation is also attached to the process which corresponds to the functional goal.

\section{9) Delete Expectation (DE)}

Delete the expectation corresponding to the nonfunctional goal, which is deleted by end-user.

\section{Case Study}

A case on planning travel is studied to illustrate the method proposed in this paper. In this case, the goal model in Figure 4 is used as the domain model.

This case belongs to the domain of urban traffic, and takes common citizens' travel as background. Although common citizens travel every day, they are not experts on the domain of urban traffic. They are assumed to propose requirements in the form of goals, and their requirements are usually not complete and correct. Some of the goals even do not belong to the domain of urban traffic. We should analyze the requirements with those characteristics, and assist them to customize the software suitable for them with Web services. Firstly, an end-user has proposed the following goals.

1) Functional Goal: Inquire Travel Information.

2) Functional Goal: Display Travel Information by E-map. 


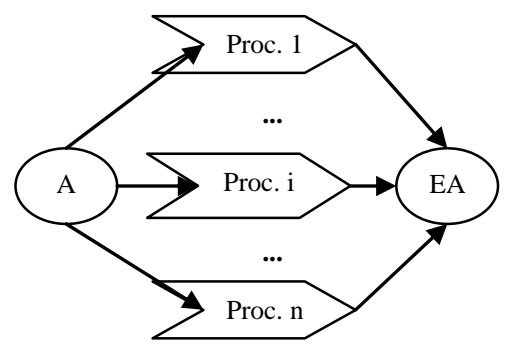

a

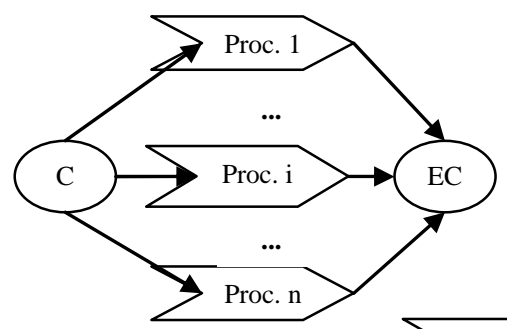

c

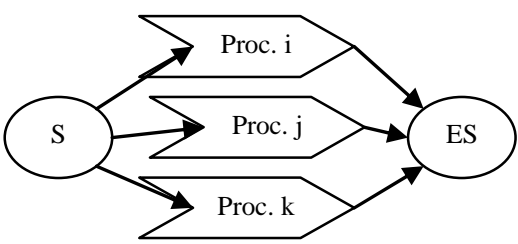

f

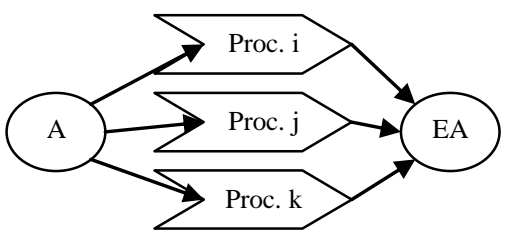

b

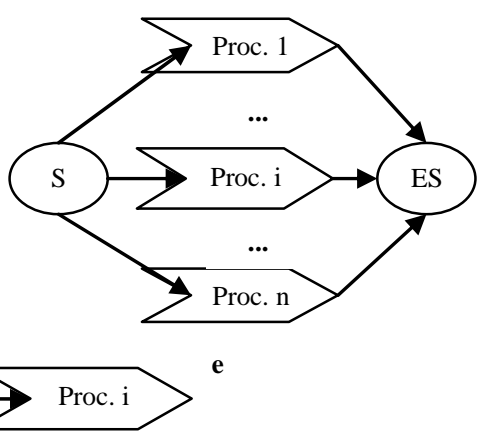

g

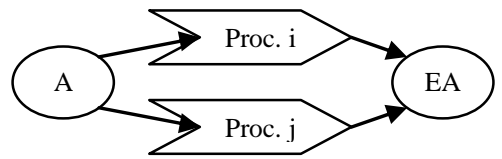

$\mathbf{h}$

Figure 3. Personalized process customization.

3) Functional Goal: Plan Itinerary of Bus.

4) Qualitative Nonfunctional Goal: Number of Itinerary/ Numerous, which constrains Plan Itinerary.

5) Functional Goal: Search Gas Station.

We will perform personalized operation and correctness detection based on the requirements proposed above.

The end-user feels "Plan Itinerary" should have nonfunctional goal "Response Time/Short", which triggers "check conflict", and finds that "Number of Itinerary/ Numerous" is conflicted "Response Time/Short". We suggest the end-user to do some modification. Finally, the end-user changes the "Number of Itinerary/Numerous" into "Number of Itinerary/Moderate".

At this time, the end-user feels that "Plan Itinerary of Bus" can not express what he/she wants to do, so he/she specifies it into "Plan Itinerary of Bus by Current Position". The personalized operation "specify goal” does not trigger any correctness detection.

Because the goal "Search Gas Station" depends on the goal "Confirm Current Position", the end-user feel it is necessary to add "Confirm Current Position" to complete the requirements. "Check conflict" is triggered.
The end-user wants to add a goal "Inquire Weather" which belongs to another domain, and no correctness detection is triggered.

The end-user feels he/she does not need the goal "Inquire Travel Information" any more. After deleting the goal, "Check Nonfunctional Goal Collection” and "Check Sub-goal Collection” are triggered.

After the personalized operations performed above, we get the personalized goal model. Using process customization rules "DPA" and "APA", we get the personalized process (Figure 5). The composite process "Display Travel Information" and "Plan Itinerary" can also be customized similarly according to the rules.

The personalized processes are the basis for service discovery and composition.

\section{Prototype System}

A prototype tool for service-oriented requirements elicitation and analysis is developed to support the method discussed above. Its functions include requirements elicitation, requirements analysis, and service discovery, composition, and execution.

The tool is B/S style to satisfy multi-users online, and 

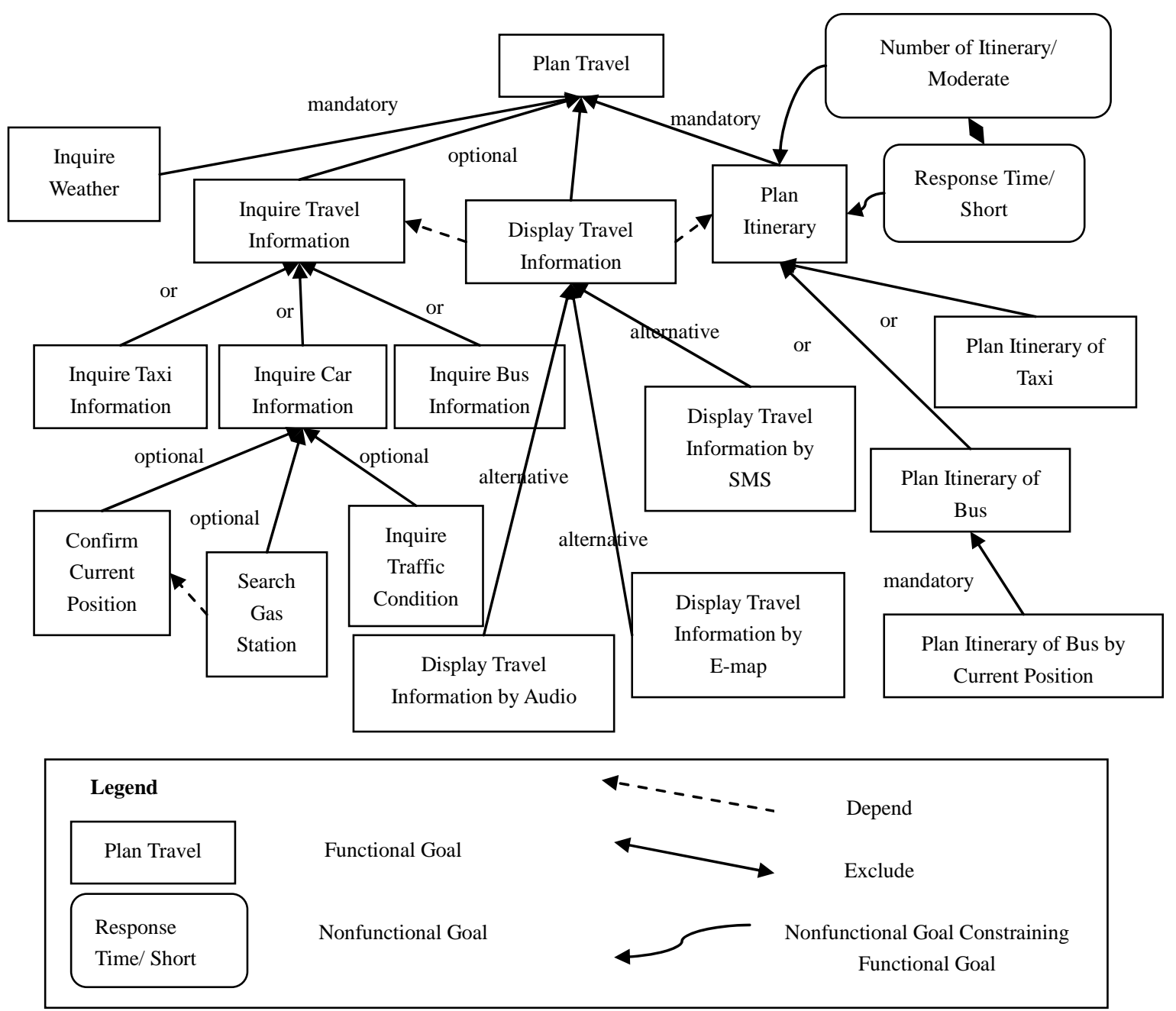

Figure 4. Goal model on traffic.

Composite Process in Domain Model:

Plan Travel

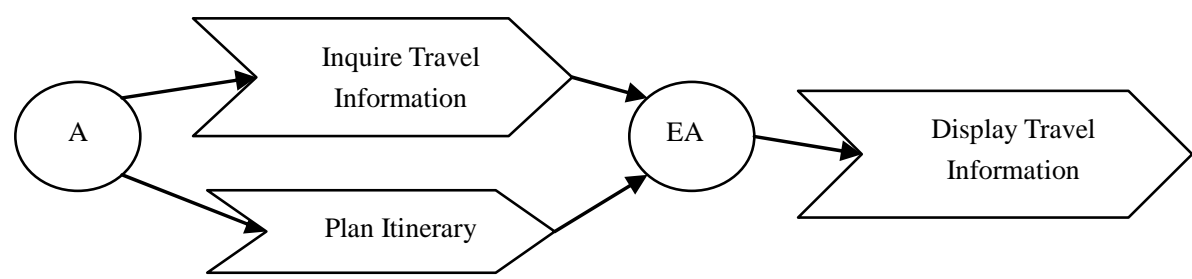

Personalize Process:

Plan Travel

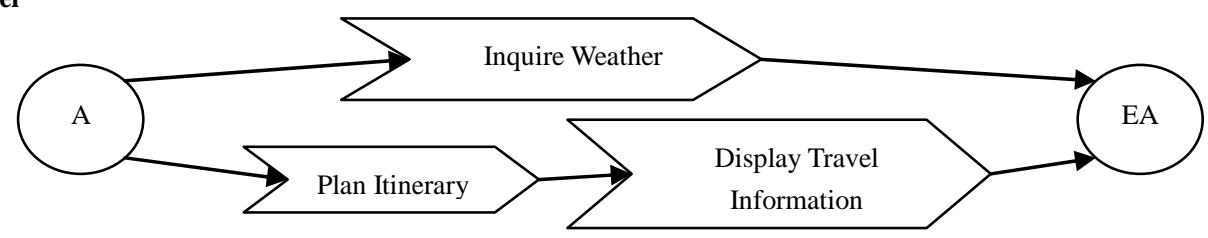

Figure 5. Personalized process customization. 


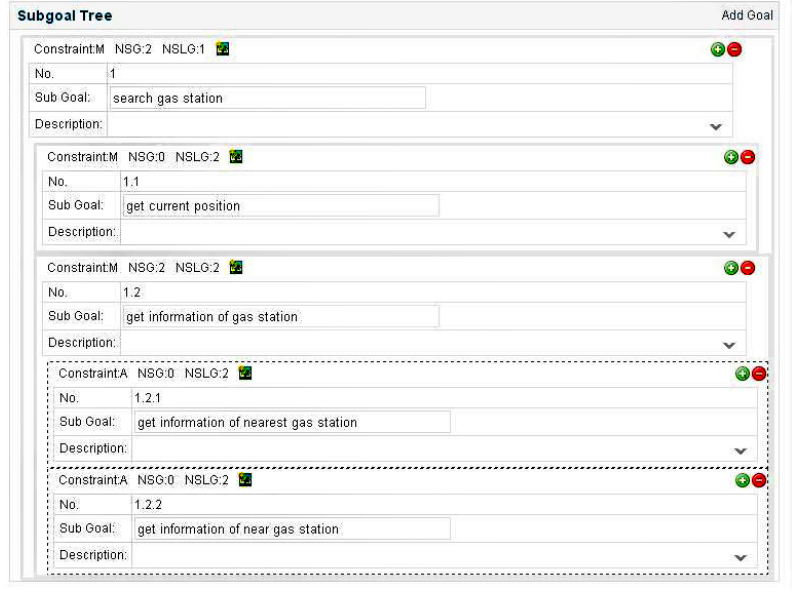

Figure 6. the Interface for end-users' personalized operations.

is implemented in the form of Web service.

End-users can register to get an account. They can input initial requirements by structured natural language after login. After analyzing the requirements, we can get a set of initial goals. End-users can perform personalized operations on the goals. The tool will detect correctness of the requirements. When the end-user finishes personalized operations, the tool can assist end-user to get personalized processes according to the goal models. Web service discovery, composition and execution are also functions of the tool, but the methods supporting those functions are not in the scope of this paper. By the way, end-users can reuse others' requirements directly, or perform personalized operations on others' requirements to generate a new version of requirements in our tool.

Figure 6 illustrates the interface for end-users' personalized operations. This interface contains some basic information of goals and supports adding, deleting and modifying those goals.

This tool has been applied in the domain of urban traffic. Our methods are proved workable in this domain. We will further apply this tool in other domains.

\section{Conclusions and Future Works}

Towards the characteristics of requirements engineering in the era of service computing, we propose a method for service-oriented requirements elicitation and analysis. Our method is faced to end-users, who have some basic knowledge about how to use computer and Internet. Requirements' correctness detection and personalized process customization are two core parts of our method, which are driven by end-users' personalized operations. We categorize the personalized operations and define the corresponding detection type and process customization rules for each type of personalized operation. A proto- type tool is illustrated to prove the method workable.

In the future, we will consider the environment of cloud computing, especially process dynamic generation and management in multi-tenant environment.

\section{Acknowledgements}

This research is part of the projects which are supported by the National Basic Research Program (973 Program) of China (2007CB310801); the National Natural Science Foundation of China (60970017, 60703009, 60940028); the Scholarship for International Young Scientists funded by National Natural Science Foundation of China (60950110352); Specialized Research Fund for the Doctoral Program of Higher Education (20090141120020); Fundamental Research Funds for the Central Universities (Wuhan University) (6082008); the Outstanding Youth Foundation of Hubei Province (2009CDA148); the Youth Chenguang Science Project of Wuhan (200950431189).

\section{REFERENCES}

[1] U. Wahli, L. Ackerman, A. D. Bari, et. al., "Building SOA Solutions Using the Rational SDP,” IBM Redbook, 2007. http://www.redbooks .ibm.com.

[2] K. Q. He, R. Peng, W. Liu, J. Wang and B. Li, "Networked Software,” In Chinese, Science Press, Beijing, 2008.

[3] K. Q. He, J. Wang and P. Liang, "Semantic Interoperability Aggregation in Service Requirements Refinement," Journal of Computer Science and Technology (JCST), Vol. 25, No. 6, 2010, pp. 1103-1117. doi:10.1007/ s11390-010-9392-3

[4] K. Q. He, P. Liang, J. Liu, et al., "Design Methodology of Networked Software Evolution Growth Based on Software Patterns," Journal of System Science and Complexity, Vol. 19, No. 2, 2006, pp. 157-181. doi: 10.1007/ s11424-006-0157-6

[5] P. Liang, P. Avgeriou, K. Q. He and L. Xu, "From Collective Knowledge to Intelligence: Pre-Requirements Analysis of Large and Complex Systems,” Proceedings of the 1st Workshop on Web 2.0 for Software Engineering (Web2SE),” Cape Town, 2010, pp. 26-30.

[6] F. He, "Yoshiaki Fukazawa: Requirement Life Cycle and its Integrated Modeling Framework," Special Issue on Software Engineering and Complex Networks, Dynamics of Continuous, Discrete and Impulsive Systems, Series B, Applications \& Algorithms, Watam Press, Canada, Vol. 14(S6), 2007, pp. 227-232

[7] K. C. Kang, S. G. Cohen, J. A. Hess et al., "FeatureOriented Domain Analysis (FODA) Feasibility Study," Technical Report CMU/SEI-90-TR-21, Software Engineering Institution, Carnegie Mellon University, Pittsburgh, 1990.

[8] K. C. Kang, J. Lee and P. Donohoe, "Feature-Oriented Product Line Engineering,” IEEE Software, Vol. 19, No. 4, 2002, pp. 58-65. doi:10.1109/MS.2002.1020288 
[9] J. Mylopoulos, L. Chung and E. Yu, "From Object-Oriented to Goal-Oriented Requirements Analysis," Communications of the ACM, 1999, Vol. 42, No. 1, pp. 31-37. doi:10.1145/291469.293165

[10] A. Dardenne, A. van Lamsweerde and S. Fickas," GoalDirected Requirements Acquisition," Science of Computer Programming, Vol. 20, 1993, pp. 3-50. doi:10.1016/ 0167-6423(93)90021-G

[11] P. Bresciani, A. Perini, P. Giorgini, F. Giunchiglia and J. Mylopoulos, "Tropos: An Agent-Oriented Software Development Methodology,” Autonomous Agents and Multi-Agent Systems, Vol. 8, No. 3, 2004, pp. 203-236. doi:10. 1023/B:AGNT.0000018806.20944.ef

[12] L. Penserini, A. Perini, A. Susi and J. Mylopoulos, "From Stakeholder Needs to Service Requirements," Proceedings of International Workshop on Service-Oriented Computing: Consequences for Engineering Requirements (SOCCER'06) at 14th IEEE International Conference on Requirements Engineering (ICRE 2006), Minneapolis, 2006.

[13] J. Cao, S. S. Zhang and M. L. Li, “A Goal Driven and Process Reuse Based Web Service Customization Model," Chinese Journal of Computers, In Chinese, Vol. 28, No. 4, 2005, pp. 721-730.

[14] C. Rolland, C. Souveyet and C. BenAchour, "Guiding Goal Modeling Using Scenarios,” IEEE Transactions on
Software Engineering, Vol. 24, No. 12, 1998, pp. 10551071. doi:10.1109/32.738339

[15] Service Oriented Architectures for All (SOA4All). http://www.soa4all.eu/

[16] K. Q. He, Y. F. He, C. Wang, P. Liang and J. Liu. "Ontology \& Meta-Modeling Theory and Methodology with Its Applications,” In Chinese, Science Press, Beijing, 2008.

[17] W3C, OWL Web Ontology Language Overview, 2004. http://www.w3.org/TR/owl-features/

[18] W3C. OWL-S: Semantic Markup for Web Services, 2004. http://www.w3.org/Submission/ OWL-S/

[19] J. Wang, K. Q. He, P. Gong, C.Wang, R. Peng and B. Li, "RGPS: A Unified Requirements Meta-Modeling Frame for Networked Software," Proceedings of Third International Workshop on Advances and Applications of Problem Frames (IWAAPF 2008) at 30th International Conference on Software Engineering (ICSE 2008), Leipzig, 2008, pp. 29-35.

[20] Z. X. Sun, J. Wang, K. Q. He, S. J. Xiang and D. H. Yu, "A Model Transformation Method in Service-Oriented Domain Modeling," Proceedings of 21st Australasian Software Engineering Conference (ASWEC 2010)," Auckland, 2010, pp. 107-116. doi:10.1109/ASWEC. 2010.32 